have demonstrated the efficacy of HPV quadrivalent vaccine also for men in preventing external genital warts.

The situation of availability and reimbursability of HPV vaccine is different among countries. In Italy vaccination is free of charge for girls aged 12-14 years and is available at a special prize (about $50 €$ per shot) for women up to 45 . Men should pay the whole some of about $200 €$ per shot.

Methods We present the result of a questionnaire on the acceptability of Tetravalent vaccine among male population attending the STD Centre of Milan, particularly focused on the price patient could afford for the vaccine

Conclusion Our study demonstrated that the price of the vaccine greatly affect the acceptability. At a social price of $50 €$ per shot only a low percentage of patient would undergo the vaccination.

\section{P3.370 DAILY ORAL EMTRICITABINE/TENOFOVIR PRE-EXPOSURE PROPHYLAXIS AND PREVENTION OF HSV-2 ACOUISITION AMONG HETEROSEXUAL MEN AND WOMEN}

doi:10.1136/sextrans-2013-051184.0823

${ }^{1}$ C Celum, ${ }^{2} \mathrm{R}$ Morrow, ${ }^{2} \mathrm{D}$ Donnell, ${ }^{1} \mathrm{~T}$ Hong, ${ }^{1} \mathrm{~K}$ Thomas, ${ }^{3 \mathrm{~K}}$ Fife, ${ }^{4} \mathrm{E}$ Nakku-Joloba, ${ }^{1} \mathrm{~A}$ Mujugira, 'J Baeten. 'University of Washington, Seattle, WA, United States; ${ }^{2}$ Fred Hutchinson Cancer Research Center, Seattle, WA, United States; IIndiana University, Indianapolis, IN, United States; 'Makerere University, Kampala, Uganda

Background Daily oral and pericoital tenofovir-based antiretroviral pre-exposure prophlyaxis (PrEP), reduced risk of HIV-1 acquisition in trials among high risk populations; oral emtricitabine(FTC)/ tenofovir (TDF) received a label indication for HIV-1 prevention. Peri-coital dosing of $1 \%$ tenofovir gel also reduced the risk of acquisition of herpes simplex virus type 2 (HSV-2), by $51 \%$. Tenofovir has anti-HSV-2 activity in vitro, although the EC90 is high. We conducted a randomised, placebo-controlled trial of daily oral TDF and FTC/TDF as PrEP among 4747 HIV-1 uninfected partners in of heterosexual serodiscordant partnerships from Kenya and Uganda (the Partners PrEP Study) and demonstrated FTC/TDF reduced HIV-1 acquisition by $75 \%$. We performed a secondary analysis of HSV-2 acquisition among initially HSV-2 seronegative participants.

Methods We tested archived sera from baseline for HSV-2 by EIA (Focus) and HSV-2 Western blot. Among initially HSV-2 seronegative participants, HSV-2 seroconversion was assessed at the last study visit prior to unblinding the placebo arm in July 2011.

Results 528 participants on FTC/TDF (35\%) and 481 participants on placebo (32\%) were HSV-2 seronegative at baseline. Eighty-nine post-randomization HSV-2 seroconversions were observed: 37 among those assigned FTC/TDF (incidence 4.4/100 person-years) and 52 among those assigned placebo (incidence 6.6/100 personyears). Compared to placebo, FTC/TDF reduced HSV-2 acquisition by $35 \%$ (95\% CI 1 to $58, p=0.05)$. Anti-HSV-2 effects trended towards protection for both men and women. Case-cohort analysis of plasma tenofovir levels to determine efficacy by drug exposure and subgroup analysis by HSV-2 status of the HIV-1 infected partner are underway.

Conclusions Among African heterosexual men and women, daily oral FTC/TDF PrEP modestly reduced the risk of HSV-2 infection in the context of a study population with high adherence and for whom high efficacy against HIV-1 acquisition was demonstrated. Potential protection against HSV-2 in addition to HIV-1 could increase the public health benefits of PrEP.

\section{P3.371 REDUCTION IN HPV PREVALENCE AMONG YOUNG WOMEN FOLLOWING INTRODUCTION OF HPV VACCINE IN THE UNITED STATES AND ESTIMATED VACCINE EFFECTIVENESS}

doi:10.1136/sextrans-2013-051184.0824
L E Markowitz, 'S Hariir, 'C Lin, 'E Dunne, 'M Steinau, ${ }^{2} \mathrm{G}$ McQuillan, ${ }^{1} \mathrm{E}$ R Unger. ${ }^{1}$ Centers for Disease Control and Prevention, Atlanta, GA, United States, ${ }^{2}$ Centers for Disease Control and Prevention, Hyattsville, MD, United States

Background Human papillomavirus (HPV) vaccination was introduced into the adolescent immunisation schedule in the United States in mid 2006. Vaccination is recommended for females at age 11 or 12 years and through age 26 if not previously vaccinated. Estimated 3-dose coverage was 32\% among 13-17 year-olds in 2010.

Objectives To compare HPV prevalence among females in the first 4 years of the vaccine era (2007-2010) with the prevaccine era (2003-2006), and to determine vaccine effectiveness (VE).

Methods The National Health and Nutrition Examination Surveys (NHANES) are a series of cross sectional surveys, designed to be nationally representative of the civilian, non-institutionalised US population. HPV prevalence was determined in self-collected cervicovaginal swabs from females aged $14-59$ years; 4150 in 2003-2006 and 4253 in 2007-2010. Type-specific HPV prevalence was determined by the Linear Array HPV Genotyping Assay. VE was estimated among sexually active 14-26 year-olds in 2007-2010.

Results Among females aged 14-19 years, vaccine type (VT) HPV prevalence decreased from $11.5 \%(95 \% \mathrm{CI}=9.2,14.4)$ in $2003-2006$ to $5.1 \%(95 \% \mathrm{CI}=3.8,6.6)$ in $2007-2010$; a $56 \%(95 \% \mathrm{CI}=37 \%$, 69\%) decline

Prevalence did not differ between the two time periods in other age groups. History of vaccination was associated with lower VT HPV prevalence among sexually active $14-19$ years-olds, $3.5 \%$ vs. $12.6 \%(\mathrm{aRR}=0.18 ; 95 \% \mathrm{CI}, 0.7-0.48$, estimated $\mathrm{VE}=82 \%)$ and among $20-26$ year-olds, $12.4 \%$ vs $21.3 \%$ (aRR $=0.46$; $95 \%$ CI, 0.22 0.99 , estimated $\mathrm{VE}=54 \%$ ). Our sample size was too small to evaluate effectiveness by number of doses.

Conclusions Within 4 years of vaccine introduction, there was a decrease in VT HPV prevalence in a nationally representative sample of females aged 14-19 years. As expected, VE was lower among those vaccinated at older ages. Ongoing monitoring will allow assessment of vaccine impact on prevalence, possible cross protection or type replacement.

\section{P3.372 SYSTEMATIC REVIEW AND META-ANALYSIS OF L1-VLP- BASED HUMAN PAPILLOMAVIRUS VACCINE EFFICACY AGAINST ANOGENITAL PRE-CANCER IN WOMEN WITH EVIDENCE OF PRIOR HPV EXPOSURE}

\author{
doi:10.1136/sextrans-2013-051184.0825
}

A Miltz, H Price, M Shahmanesh, A Copas, R Gilson. University College London, London, UK

Background It is unclear whether L1-VLP-based human papillomavirus (HPV) vaccines are efficacious in preventing anogenital precancer in women with prior vaccine-type HPV exposure. Participants in the phase III efficacy trials were not excluded if infected at baseline (HPV-DNA and serology were performed in retrospect); the efficacy in this sub-group of vaccinees can be derived from published reports.

Methods A systematic review and meta-analysis was conducted to compare the efficacy of L1-VLP-based HPV vaccines with control (hepatitis A or placebo). Randomized-controlled trials (including post-RCT follow-on cohort studies) were identified from MEDLINE, Embase, Web of Science ${ }^{S M}$, PubMed, Cochrane (and quoted references). Three vaccines were evaluated: Cervarix ${ }^{\mathrm{TM}}$ containing HPV-16/18 VLPs (GSK), Gardasil ${ }^{\circledR}$ containing HPV-6/11/16/18 VLPs (Merck), and an HPV-16 monovalent vaccine (Merck Research Laboratories).

Results Three RCT reports and one post-RCT follow-on study met the eligibility criteria, comprising data from 13,339 women who were included in the vaccine studies but had evidence of HPV 